\title{
Intracranial giant cell tumour-a case report
}

\author{
Hilwati Hashim ${ }^{1 *}$ (D) and Nor Salmah Bakar ${ }^{2}$
}

\begin{abstract}
Background: Giant cell tumours (GCT) are rare benign bone tumours that typically occur in the appendicular skeleton. In typical locations, these tumours are relatively simple to diagnose by radiography. However, at atypical locations, these tumours can be confused with other disease pathologies. Here, we describe a patient with an intracranial GCT that was initially thought to be a cystic teratoma.

Case presentation: A 49-year-old man with no previous medical illness was investigated for left-sided hearing impairment and tinnitus associated with a left-sided headache of 2 years' duration. He did not complain of fever, vertigo, nasal or constitutional symptoms. Examination confirmed a sensory neural hearing loss with no other neurological deficit. Imaging by CT and subsequent MRI revealed an extra axial, multiloculated, and septated mass at the left temporal area with a significant mass effect. A provisional diagnosis of cystic teratoma was made. However, histopathological examination of the resected mass indicated that the lesion was a GCT. The patient required a second excision of the tumour, which he refused. His latest follow-up MRI showed no further progression of the residual tumour. His headaches have resolved and there was no progression of other symptoms.

Conclusion: This case highlights that GCTs can occur at sites outside the appendicular skeleton and that these tumours may be confused with other diseases that have a similar appearance on imaging.

Keywords: Giant cell tumours, Skull, CT scan, MRI scan, Case report
\end{abstract}

\section{Background}

Giant cell tumours (GCT) are rare benign tumours that typically occur at long bones [1]. When these tumours occur at intracranial sites, they can be confused with other conditions, particularly since the frequency of GCTs at these locations is low. Although benign in nature, these tumours can be locally aggressive and affect adjacent, critical structures [1]. Here, we describe a rare case of intracranial GCT and accompanying radiological and histological findings. This case serves to emphasize the challenges associated with recognizing these tumours and providing a differential diagnosis based on radiology findings.

\footnotetext{
*Correspondence: hilwa167@uitm.edu.my

'Department of Radiology, Faculty of Medicine, Universiti Teknologi MARA, Sungai Buloh, Malaysia

Full list of author information is available at the end of the article
}

\section{Case presentation}

A 49-year-old male was referred to our neurosurgical clinic where he complained of left sided hearing impairment, left ear tinnitus, and left-sided headache of 2 years' duration. These symptoms were not associated with vertigo, fever, nasal symptoms, or ear discharge. His neurological examinations were normal. Examination of the external auditory canal (EAC) showed EAC stenosis that limited visualization of the tympanic membrane. Pure tone audiometry (PTA) confirmed a profound sensoryneural hearing loss on the left side. The right ear examination was normal.

Contrasted computed tomography (CT) and subsequently magnetic resonance imaging (MRI) of the brain showed a large, peripherally enhancing, multi-septated, left temporal fossa extra-axial lesion that caused thinning of the left temporo-parietal bone and destruction of left temporal bone and mastoid air cells. Mass effect and 
midline shift were also visible. Contrasted computed tomography $(\mathrm{CT})$ and subsequent magnetic resonance imaging (MRI) of the brain showed a large, peripherally enhancing, multi-septated, left temporal fossa extra-axial lesion that caused thinning of the temporo-parietal bone and temporal bone destruction on the left side. Mastoid air cells with mass effect and midline shift were also visible on the left side. On MRI, the lesion was heterogeneously hyperintense on T1-weighted images (T1W), suggestive of fluid with proteinaceous content. Furthermore, the lesion was hyperintense on T2-weighted images (T2W) and had a partially suppressed FLAIR sequence that correlated with the proteinaceous fluid noted on T1W. A thick-wall peripheral enhancement was seen after administration of the contrast agent (Figs. 1 and 2). Correlating with the patient's history and physical examination, the reporting radiologist considered a provisional diagnosis of cystic teratoma. Surgery was scheduled for this patient one week after the examination. In the interim, the patient was treated with intravenous ceftriaxone to treat a possible intracranial infection.

Intra-operatively, the squamous part of the left temporal bone appeared to be paper-thin. Upon removal of the bone flap, the inner table of the temporal bone had a ragged margin, which suggested possible tumour invasion. A well-encapsulated, extra-dural lesion containing "cheese-like" material and greenish oily fluid occupied the left temporal fossa. Central decompression of the tumour was done followed by peripheral separation of the capsule from the dura. However, the surgeons could not completely remove the tumour as it was medially adherent to the base of skull. Thus, the medial part of the tumour was coagulated and left in place.

Histopathological examination of the tumour showed proliferation of stromal cells mixed with varying numbers of multinucleated giant cells that exhibited vesicular nuclei with prominent nucleoli, similar to those seen in stromal cells. The stroma cells were mononuclear and formed vague, focal fascicular arrangements. These cells had round, oval, or spindle-like nuclei. Macrophage-like cells were also present (Fig. 3). The presence of characteristic features of mononuclear and multinucleated giant cells confirmed the diagnosis of GCT. The absence of cellular atypia and abnormal mitoses supported a benign morphology.

The post-operative recovery of this patient was uneventful and he was discharged 1 week after surgery. A follow-up MRI showed residual tumour bulk. Further debulking of the tumour followed by an oncology referral was planned for the patient. However, he declined further management as his symptoms, apart from hearing loss, had resolved. He did agree to continue followup with the neurosurgical team and was subsequently fitted with a hearing aid. Three years later, the residual tumour showed no further growth and the patient remained well with no new symptoms.

\section{Discussion}

GCTs account for approximately $5 \%$ of all bone tumours and $20 \%$ of all benign bone tumours. There is a slight female preponderance for these tumours and most cases are diagnosed when patients are between 20 and 44 years old [2]. GCTs typically occur at the distal femur, the

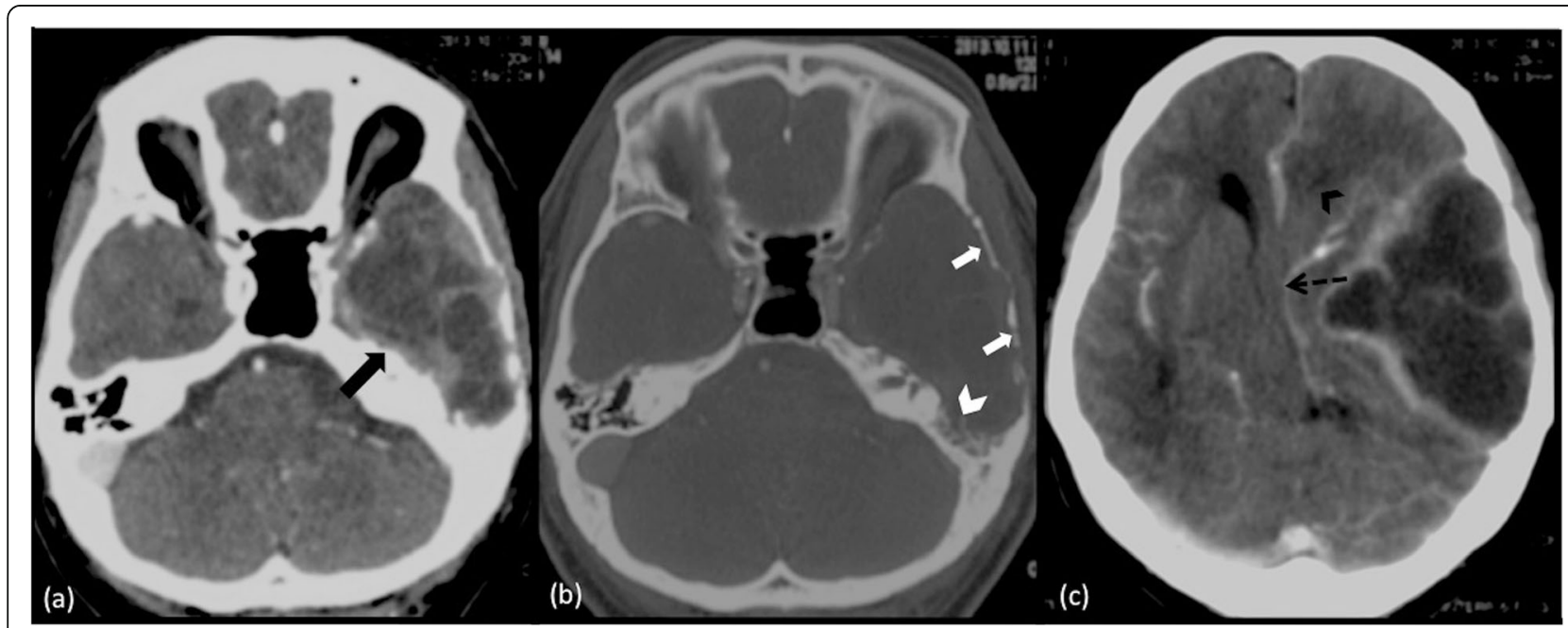

Fig. 1 Contrasted CT of the brain in the axial plane. a An extra-axial, peripherally enhancing multiseptated lesion is seen at the left temporal fossa (black arrow). b The lesion caused thinning of the temporal bone (white arrow) with destruction of the petrous temporal bone (arrowhead). c The lesion extended to the left parietal area and caused compression of the left lateral ventricle (black arrowhead) as well as a midline shift to the right (broken arrow) 


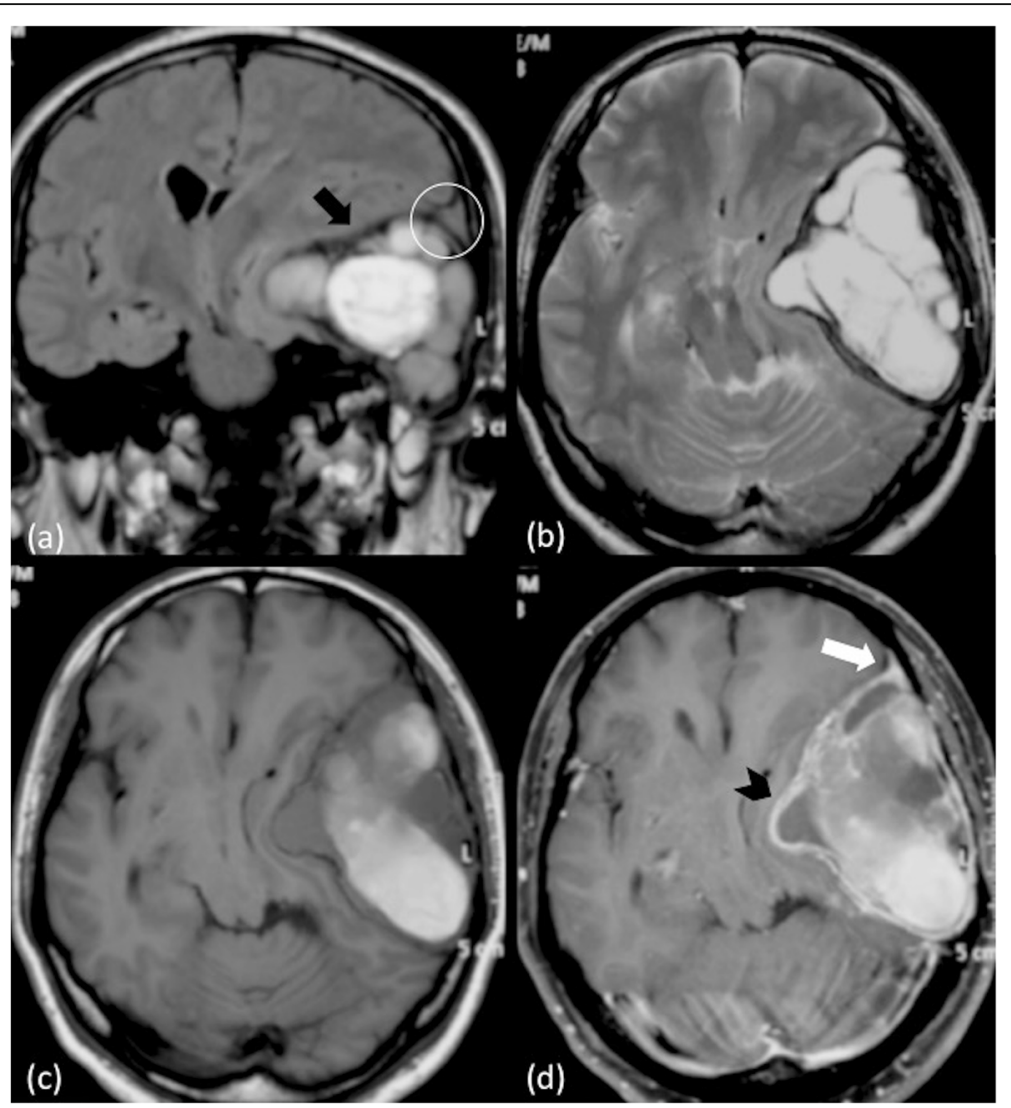

Fig. 2 Contrasted MRI of the brain. a Coronal FLAIR. b Axial T2-weighted. c Axial T1-weighted. d Axial post-contrast T1-weighted images. Coronal FLAIR showed that the tumour occupied the left temporal fossa (black arrow). The tumour was hyperintense on T2-weighted images (b), heterogeneously hyperintense on T1-weighted images (c), and had thick-wall peripheral enhancement (black arrowhead) in the post contrast T1weighted sequence (d). The presence of a CSF cleft (circled) and dural tail (white arrowhead) suggested that the tumour is extra-axial

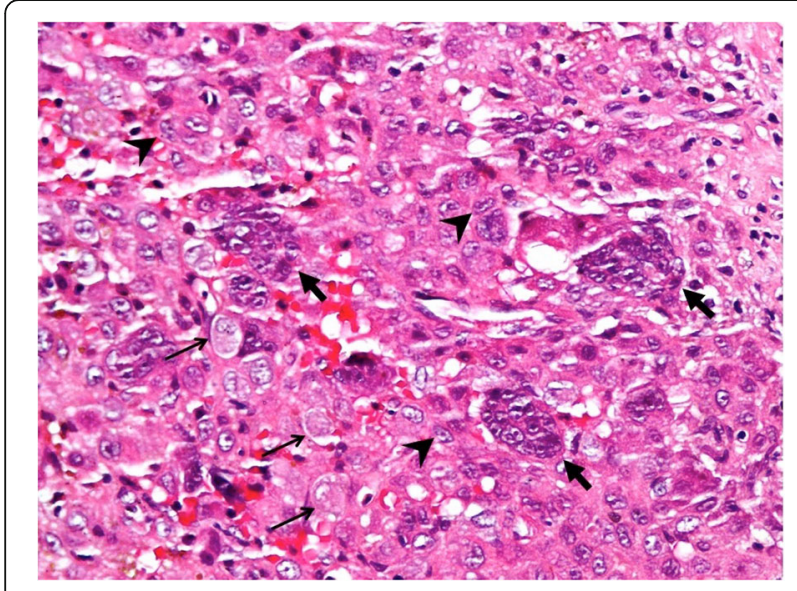

Fig. 3 H\&E staining at $\times 400$ magnification shows proliferation of stromal mononuclear cells mixed with variable numbers of multinucleated giant cells (arrows). The multinucleated giant cells exhibit vesicular nuclei with prominent nucleoli that are similar to those seen for stromal cells (mononuclear stromal cells are present between multinucleated giants with several examples of stromal cells indicated by arrowheads). Rounded macrophage-like cells can also be seen (thin arrows) proximal tibia, the distal radius, and the sacrum and are particularly frequent at the long bone epiphyses [1]. Anecdotal case reports of cranial GCTs describe tumours that arise from sphenoid, temporal, and occipital bones of the skull and frontal sinus [3-5]. In our patient, the tumour likely arose from the left temporal fossa, specifically the left temporal bone, as was seen intra-operatively.

GCTs develop by endochondral ossification. Sphenoid and temporal bones also develop from endochondral ossification, which can explain the preponderance of cranial GCTs arising at these bones [4]. Although GCTs are benign tumours, they have varying degrees of aggressiveness. Pulmonary metastases have been reported. Three cells types comprise GCTs: multinucleated giant cells, mononuclear cells with a round morphology resembling monocytes or macrophage-like cells, and mononuclear stromal cells that may show round, oval, or spindle-shaped nuclei [6]. Mononuclear stromal cells are neoplastic cells that may show cellular atypia in malignant counterparts of GCTs, although malignant GCTs are very uncommon. In the histopathological examination conducted for this patient, all three cell types were observed. 
The clinical presentation of GCTs depends on the tumour location. Temporal bone GCTs can cause hearing loss and facial paresis [7, 8]. These tumours grow slowly and thus patients typically have a long-standing history.

GCTs of the long bones have no characteristic features on radiographs, although they often have a radiolucent appearance at the epiphyseal end of the bone [1]. However, for cranial GCTs, skull radiographs are not commonly taken given that these images are typically not useful for the investigation of a space-occupying lesion or, as in the case of this patient, hearing loss. Matushita et al. [5] described a frontal sinus GCT in which the radiograph showed osteolytic lesions in the frontal sinuses with surrounding wall destruction, a finding that suggested an aggressive lesion that could be a malignancy or osteomyelitis, but was not specific for GCT. Cross-sectional imaging of the brain also produces nonspecific findings for GCT. Most previous studies described CT imaging showing an expansive lesion causing bone destruction $[4,5,7,9]$. Such lesions have variable signal intensity and enhancement on MRI, depending on the amount of necrosis, soft tissue, or bony components present $[4,7]$. However, CT and MRI are useful methods to evaluate the extent of bony destruction and intracranial involvement, respectively.

As GCTs have no characteristic imaging features, many differential diagnoses are often considered including eosinophilic granuloma, plasmacytoma, metastatic lesion, fibrous dysplasia, brown tumour of hyperparathyroidism, or osteomyelitis [4, 7-9]. In our patient, cystic teratoma was considered since teratomas can have variable signal intensity on MRI that reflects varying components of these tumours. Based on the hearing loss for this patient as well as lesion density and signal behaviour on MRI, atypical location of a cholesterol granuloma could also be included in the differential diagnosis. This range of differential diagnoses exemplifies the difficulty in recognizing intracranial GCTs based on imaging findings. Thus, the final diagnosis of GCTs should rely on histopathological examination to demonstrate the presence of multinucleated giant cells, mononuclear cells resembling monocytes, and spindle-shaped stromal cells.

Complete surgical resection is the treatment of choice for GCTs occurring in the long bones [7, 8]. However, complete surgical resection can be difficult to achieve for cranial tumours, as demonstrated for this case. Prognosis for GCTs depends on the extent of the surgical resection [9]. Adjuvant treatment with radiation therapy has been used but remains controversial as this method does not seem to alter outcomes [7]. GCTs can have malignant potential, typically lung metastases. Our patient had significant residual disease that required further resection and subsequent referral for radiation therapy. He declined both treatments. Follow-up imaging through 4 years after initial treatment showed that the residual tumour was stable and there was no further progression of symptoms.

\section{Conclusion}

GCTs are rare, particularly those arising in the skull. Thus, GCTs are typically not considered among differential diagnoses of intracranial tumours. The lack of specific imaging findings can contribute to the confusion, and final diagnosis of GCTs can only be achieved with histology evaluation. This case serves to raise awareness about GCTs and consideration of this tumour type in the differential diagnosis of skull tumours, particularly those arising from sphenoid or temporal bones.

\section{Abbreviations}

EAC: External auditory canal; PTA: Pure tone audiometry; CT: Computed tomography; MRI: Magnetic resonance imaging; T1W: T1-weighted; T2W: T2weighted; GCT: Giant cell tumour

\section{Acknowledgements}

Department of Neurosurgery and Department of Radiology, Sungai Buloh Hospital, Malaysia. The authors would also like to thank the Director of Health Malaysia for permission to publish this paper.

\section{Authors' contributions}

$\mathrm{HH}$ and NSB contributed to the conception, acquisition, and interpretation of data, participated in the drafting of the article, and involved in the final version submitted. All authors have read and approved the manuscript.

\section{Funding}

This study is supported by a research grant from Universiti Teknologi MARA ref.no 600-IRMI/DANA KCM 5/3/LESTARI (209/2017).

\section{Availability of data and materials}

Data sharing is not applicable to this article as no datasets were generated or analysed during the current study.

Ethics approval and consent to participate

Not applicable.

\section{Consent for publication}

The patient included in this case report gave written informed consent for this case to be published.

\section{Competing interests}

None

\section{Author details}

${ }^{1}$ Department of Radiology, Faculty of Medicine, Universiti Teknologi MARA, Sungai Buloh, Malaysia. ${ }^{2}$ Centre for Pathology Diagnostic and Research Laboratories, Faculty of Medicine, Universiti Teknologi MARA, Sungai Buloh, Malaysia.

Received: 26 April 2020 Accepted: 7 September 2020

Published online: 15 September 2020

\section{References}

1. Sobti A, Agrawal P, Agarwala S, Agarwal M (2016) Giant cell tumor of bone an overview. Arch Bone Jt Surg. 4(1):2-9

2. Beebe-Dimmer JL, Cetin K, Fryzek JP, Schuetze SM, Schwartz K. (2009) The epidemiology of malignant giant cell tumors of bone: an analysis of data from the Surveillance, Epidemiology and End Results Program (1975-2004). Rare Tumors. (2):e52

3. Company M, Ramos R. Giant cell tumor of the sphenoid. (2009) Archives of Neurology:66(1):134-135. 
4. Lu ZH, Yao ZW (2011) Giant cell tumour of the posterior cranial fossa: a case report. Brit J Radiol 84(1007):e208-ee11

5. Matushita JP, Matushita JS, Simões LAM, Carvalho Neto LFd, Matushita CS, Matushita Junior JPK. (2013) Giant cell tumor of the frontal sinus: case report. Radiologia Brasileira:;6:255-258.

6. Wulling M, Engels C, Jesse N, Werner M, Delling G, Kaiser E (2001) The nature of giant cell tumor of bone. J Cancer Res Clin Oncol 127(8):467-474

7. Isaacson B, Berryhill W, Arts HA (2009) Giant-cell tumors of the temporal bone: management strategies. Skull Base. 19(4):291-301

8. Pai SB, Lalitha RM, Prasad K, Rao SG, Harish K. (2005) Giant cell tumor of the temporal bone - a case report. BMC Ear, Nose and Throat Disorders. 5:8

9. Harris AE, Beckner ME, Barnes L, Kassam A, Horowitz M (2004) Giant cell tumor of the skull: a case report and review of the literature. Surgical neurology. 61(3):274-277

\section{Publisher's Note}

Springer Nature remains neutral with regard to jurisdictional claims in published maps and institutional affiliations.

\section{Submit your manuscript to a SpringerOpen ${ }^{\mathcal{O}}$ journal and benefit from:}

- Convenient online submission

- Rigorous peer review

- Open access: articles freely available online

High visibility within the field

- Retaining the copyright to your article

Submit your next manuscript at $\boldsymbol{\nabla}$ springeropen.com 